\title{
Classical and Quantum Exact Solutions for a FRW Multiscalar Field Cosmology with an Exponential Potential Driven Inflation
}

\author{
J. Socorro $\mathbb{D}^{1}{ }^{1}$ Omar E. Núñez, ${ }^{1}$ and Rafael Hernández-Jiménez ${ }^{2}$ \\ ${ }^{1}$ Departamento de Física, DCeI, Universidad de Guanajuato-Campus León, 37150 León, GTO, Mexico \\ ${ }^{2}$ School of Physics and Astronomy, University of Edinburgh, Edinburgh EH9 3FD, UK \\ Correspondence should be addressed to J. Socorro; socorro@fisica.ugto.mx
}

Received 28 September 2018; Accepted 26 November 2018; Published 4 December 2018

Academic Editor: Eugen Radu

Copyright (c) 2018 J. Socorro et al. This is an open access article distributed under the Creative Commons Attribution License, which permits unrestricted use, distribution, and reproduction in any medium, provided the original work is properly cited.

\begin{abstract}
A flat Friedmann-Robertson-Walker (FRW) multiscalar field cosmology is studied with a particular potential of the form $\mathrm{V}(\phi, \sigma)=$ $\mathrm{V}_{0} \mathrm{e}^{-\lambda_{1} \phi-\lambda_{2} \sigma}$, which emerges as a relation between the time derivatives of the scalars field momenta. Classically, by employing the Hamiltonian formalism of two scalar fields $(\phi, \sigma)$ with standard kinetic energy, exact solutions are found for the Einstein-KleinGordon (EKG) system for different scenarios specified by the parameter $\lambda^{2}=\lambda_{1}^{2}+\lambda_{2}^{2}$, as well as the e-folding function $\mathrm{N}_{\mathrm{e}}$ which is also computed. For the quantum scheme of this model, the corresponding Wheeler-DeWitt (WDW) equation is solved by applying an appropriate change of variables.
\end{abstract}

\section{Introduction}

The inflation paradigm is considered the most accepted mechanism to explain many of the fundamental problems of the early stages in the evolution of our universe [1-4], such as the flatness, homogeneity, and isotropy observed in the present universe. Another important aspect of inflation is its ability to correlate cosmological scales that would otherwise be disconnected. Fluctuations generated during this early phase of inflation yield a primordial spectrum of density perturbation [5-8], which is nearly scale invariant, adiabatic, and Gaussian, which is in agreement with cosmological observations [9].

The single-field scalar models have been broadly used to describe the primordial expansion, the most phenomenological successful are those with a quintessence scalar field and slow-roll inflation [10-19]. However, if another component is included, i.e., a multiscalar field theory, it is also possible to produce an inflationary scenario [20,21], even if the fields are noninteracting [16]. Even more the dynamical possibilities in multifield inflationary scenarios are considerably richer than in single-field models, such as in the primordial inflation perturbations analysis $[22,23]$ or the assisted inflation as discussed in [24], furthermore, the general assisted inflation as in [21]. In this sense the multiscalar fields' cosmology is an attractive candidate to explain such phenomenon.

Recent works have shown that multiscalar field models are very fruitful when studying the early stages of the universe, such as the case in [25], where the authors perform a semianalytic study of preheating in inflationary models comprised of multiple scalar fields coupled nonminimally to gravity. In [26] the authors show the sensitivity of the cosmological observables to the reheating phase following inflation driven by many scalar fields, where they find that, for certain decay rate, reheating following multifield inflation can have a significant impact on the prediction of cosmological observables.

Indeed the multiscalar field models for inflation are of interest even on most recent studies, such as the abovementioned cases; however, one of the most important features in such models is the potential associated with the scalar fields, and in many cases, the employed potentials are simple polynomial powers of the scalar fields or in other cases the employed potential is a series of lineally summed 
exponentials; however, it has been shown that a potential of the form $\mathrm{V}(\phi, \sigma)=\mathrm{V}_{0} \mathrm{e}^{-\lambda_{1} \phi-\lambda_{2} \sigma}$ is a good candidate to model the inflation phenomenon for multiscalar field theory, as discussed in previous work [27], and might provide a richer postinflation scenario.

Generally, in the studies of inflationary cosmology one employs the usual slow-roll approximation with the objective to extract simple expression for basics observable, such as the scalar and tensor spectral indices, the running of the scalar spectral index, and the tensor-to-scalar ratio. Moreover, in the slow-roll regime the set of EKG equations reduces in such a way that one can quickly obtain the solution of the scale factor. Nevertheless, there is an alternative approach which allows for an easy derivation of many inflation results. It is called Hamilton's formulation, widely used in analytical mechanics. Using this method we obtain the exact solutions of the complete set of EKG equations without using the aforementioned approximation.

On the other hand, we implement a basic formulation in quantum cosmology by means of the Wheeler-DeWitt (WDW) equation. The WDW equation has been analyzed with different approaches in order to solve it, and there are several papers on the subject, such is the case in [28], where they debate what a typical wave function for the universe is. Reference [29] has a review on quantum cosmology where the problem of how the universe emerged from big bang singularity can no longer be neglected in the GUT epoch. Moreover, the best candidates for quantum solutions are those that have a damping behavior with respect to the scale factor, since only such wave functions allow for good classical solutions when using a Wentzel-Kramers-Brillouin (WKB) approximation for any scenario in the evolution of our universe [30, 31]. Furthermore, in the context of a single scalar field a family of scalar potentials is obtained in the Bohmian formalism [27, 32], where among others a general potential of the form $\mathrm{V}(\phi)=\mathrm{V}_{0} \mathrm{e}^{-\lambda \phi}$ is examined. Given this insight, for a two scalar field scenario we consider a potential of the form $\mathrm{V}(\phi, \sigma)=\mathrm{V}_{0} \mathrm{e}^{-\lambda_{1} \phi-\lambda_{2} \sigma}$ in order to solve the WDW equation.

This work is arranged as follows. In Section 2 we present the model with the action and the corresponding EKG equations for our cosmological model and the associated Hamiltonian density. In Section 3 general equations for the classical solutions of scale factor, scalar fields, and their associated momenta are derived in terms of the free parameters of the model. In Sections 3.1, 3.2, 3.3, and 3.4 the particular solutions and their number of e-folds are computed for different cases of the $\lambda$ parameter. in Section 4 we use the Hamiltonian density to compute the corresponding WDW equation, which is solved by using a change of variables; an ansatz for the wave function is employed in terms of a generic function and parameters which are to be determined. In Sections 4.1 and 4.2 the corresponding wave function and their constants relations are presented for different cases of the $\delta$ parameter, which in turn is related to the $\lambda$ parameter of the classical solutions. Finally, in Section 5 we present our conclusions for this work.

\section{The Model}

We begin with the construction of two scalar fields cosmological paradigm, which requires canonical scalar fields $\phi, \sigma$. The action of a universe with the constitution of such fields is

$$
\begin{aligned}
& \mathscr{L} \\
& =\sqrt{-\mathrm{g}}\left(\mathrm{R}+\frac{1}{2} \mathrm{~g}^{\mu \nu} \nabla_{\mu} \phi \nabla_{\nu} \phi+\frac{1}{2} \mathrm{~g}^{\mu \nu} \nabla_{\mu} \sigma \nabla_{\nu} \sigma-\mathrm{V}(\phi, \sigma)\right),
\end{aligned}
$$

where $\mathrm{R}$ is the Ricci scalar, $\mathrm{V}(\phi, \sigma)$ is the corresponding scalar field potential, and the reduced Planck mass $M_{P}^{2}=1 / 8 \pi G=$ 1. The corresponding variations of (1), with respect to the metric and the scalar fields give the Einstein-Klein-Gordon field equations

$$
\begin{aligned}
\mathrm{G}_{\alpha \beta}= & \frac{1}{2}\left(\nabla_{\alpha} \phi \nabla_{\beta} \phi-\frac{1}{2} \mathrm{~g}_{\alpha \beta} \mathrm{g}^{\mu \nu} \nabla_{\mu} \phi \nabla_{\nu} \phi\right) \\
& +\frac{1}{2}\left(\nabla_{\alpha} \sigma \nabla_{\beta} \sigma-\frac{1}{2} g_{\alpha \beta} g^{\mu \nu} \nabla_{\mu} \sigma \nabla_{\nu} \sigma\right) \\
& -\frac{1}{2} g_{\alpha \beta} V(\phi, \sigma), \\
\square \phi-\frac{\partial \mathrm{V}}{\partial \phi}= & \mathrm{g}^{\mu \nu} \phi_{, \mu \nu}-g^{\alpha \beta} \Gamma_{\alpha \beta}^{\nu} \nabla_{\nu} \phi-\left(\frac{\partial V}{\partial \phi}\right)_{\sigma}=0, \\
\square \sigma-\frac{\partial \mathrm{V}}{\partial \sigma}= & \mathrm{g}^{\mu \nu} \sigma_{, \mu \nu}-g^{\alpha \beta} \Gamma_{\alpha \beta}^{\nu} \nabla_{\nu} \sigma-\left(\frac{\partial V}{\partial \sigma}\right)_{\phi}=0 .
\end{aligned}
$$

The line element to be considered in this work is the flat FRW

$$
\mathrm{ds}^{2}=-\mathrm{N}(\mathrm{t})^{2} \mathrm{dt}^{2}+\mathrm{e}^{2 \Omega(\mathrm{t})}\left[\mathrm{dr}^{2}+\mathrm{r}^{2}\left(\mathrm{~d} \theta^{2}+\sin ^{2} \theta \mathrm{d} \phi^{2}\right)\right],
$$

where $\mathrm{N}$ is the lapse function, which in a special gauge one can directly recover the cosmic time $t_{\text {phys }}\left(\mathrm{Ndt}=\mathrm{dt}_{\text {phys }}\right)$, the scale factor $A(t)=e^{\Omega(t)}$ is in the Misner's parametrization, and the scalar function has an interval, $\Omega \in(-\infty, \infty)$. Consequently the field equations are

$$
\begin{array}{r}
\frac{3 \dot{\Omega}^{2}}{\mathrm{~N}^{2}}-\frac{\dot{\phi}^{2}}{4 \mathrm{~N}^{2}}-\frac{\dot{\sigma}^{2}}{4 \mathrm{~N}^{2}}-\frac{1}{2} \mathrm{~V}(\phi, \sigma)=0, \\
\frac{2 \ddot{\Omega}}{\mathrm{N}^{2}}+\frac{3 \dot{\Omega}^{2}}{\mathrm{~N}^{2}}-\frac{2 \dot{\Omega} \dot{\mathrm{N}}}{\mathrm{N}^{3}}+\frac{\dot{\phi}^{2}}{4 \mathrm{~N}^{2}}+\frac{\dot{\sigma}^{2}}{4 \mathrm{~N}^{2}}-\frac{1}{2} \mathrm{~V}(\phi, \sigma) \\
\frac{\ddot{\phi} \dot{\phi}}{\mathrm{N}^{2}}+\frac{3 \dot{\Omega}^{2}}{\mathrm{~N}^{2}}-\frac{\dot{\mathrm{N}} \dot{\phi}^{2}}{\mathrm{~N}^{3}}+(\dot{\mathrm{V}})_{\sigma}=0, \\
\frac{\ddot{\sigma} \dot{\sigma}}{\mathrm{N}^{2}}+\frac{3 \dot{\Omega} \dot{\sigma}^{2}}{\mathrm{~N}^{2}}-\frac{\dot{\mathrm{N}} \dot{\sigma}^{2}}{\mathrm{~N}^{3}}+(\dot{\mathrm{V}})_{\phi}=0 .
\end{array}
$$

By building the corresponding Lagrangian and Hamiltonian densities for this cosmological model, classical solutions to Einstein-Klein-Gordon equations ((2)-(4)) can be found using the Hamilton's approach, and the quantum formalism can be determined and solved. In that sense, we use the metric equation (5) into (1) having

$$
\mathscr{L}=\mathrm{e}^{3 \Omega}\left(\frac{6 \dot{\Omega}^{2}}{\mathrm{~N}}-\frac{\dot{\phi}^{2}}{2 \mathrm{~N}^{2}}-\frac{\dot{\sigma}^{2}}{2 \mathrm{~N}^{2}}+\mathrm{NV}(\phi, \sigma)\right),
$$


where upper "•" represents the first time derivative and the corresponding momenta are defined in the usual way $\Pi_{\mathrm{q}}=$ $\partial \mathscr{L} / \partial \dot{\mathrm{q}}$. We obtain

$$
\begin{aligned}
\Pi_{\Omega} & =12 \frac{e^{3 \Omega}}{N} \dot{\Omega}, \\
\dot{\Omega} & =\frac{N e^{-3 \Omega}}{12} \Pi_{\Omega}, \\
\Pi_{\phi} & =-\frac{e^{3 \Omega}}{N} \dot{\phi}, \\
\dot{\phi} & =-N e^{-3 \Omega} \Pi_{\phi}, \\
\Pi_{\sigma} & =-\frac{e^{3 \Omega}}{N} \dot{\sigma}, \\
\dot{\sigma} & =-N e^{-3 \Omega} \Pi_{\sigma} .
\end{aligned}
$$

By performing the variation of the canonical Lagrangian with respect to $\mathrm{N}$, i.e., $\delta \mathscr{L}_{\text {canonical }} / \delta \mathrm{N}=0$, where $\mathscr{L}_{\text {canonical }}=$ $\Pi_{\mathrm{q}} \dot{\mathrm{q}}-\mathrm{N} \mathscr{H}$, it implies the constraint $\mathscr{H}=0$. Hence the Hamiltonian density is

$$
\mathscr{H}=\frac{\mathrm{e}^{-3 \Omega}}{24}\left[\Pi_{\Omega}^{2}-12 \Pi_{\phi}^{2}-12 \Pi_{\sigma}^{2}-24 \mathrm{~V}(\phi, \sigma) \mathrm{e}^{6 \Omega}\right] .
$$

In the gauge $\mathrm{N}=24 \mathrm{e}^{3 \Omega}$ and using the Hamilton equations $\dot{\mathrm{q}}=\partial \mathscr{H} / \partial \Pi_{\mathrm{q}}$ and $\dot{\Pi}_{\mathrm{q}}=-\partial \mathscr{H} / \partial \mathrm{q}$, we have the following set of equations:

$$
\begin{aligned}
\dot{\Omega} & =2 \Pi_{\Omega}, \\
\dot{\phi} & =-24 \Pi_{\phi}, \\
\dot{\sigma} & =-24 \Pi_{\sigma}, \\
\dot{\Pi}_{\Omega} & =6 U \\
\dot{\Pi}_{\phi} & =\frac{\partial U}{\partial \phi}, \\
\dot{\Pi}_{\sigma} & =\frac{\partial U}{\partial \sigma},
\end{aligned}
$$

where $\mathrm{U}=24 \mathrm{~V}(\phi, \sigma) \mathrm{e}^{6 \Omega}$. Given a particular form of the potential $\mathrm{V}(\phi, \sigma)$ one can derive a relation between the time derivative of the momenta such as $\dot{\Pi}_{\phi} \propto \dot{\Pi}_{\sigma}$, provided that $\partial V / \partial \phi=\alpha \partial V / \partial \sigma$, where $\alpha$ is a constant. Such connection can be obtained considering two different configurations of the potential: $\mathrm{V}(\phi, \sigma)=\mathrm{f}\left[ \pm\left(\alpha_{1} \phi+\alpha_{2} \sigma\right)\right]$ or $\mathrm{V}(\phi, \sigma)=$ $\mathrm{V}_{1} \mathrm{f}\left[ \pm\left(\alpha_{1} \phi\right)\right]+\mathrm{V}_{2} \mathrm{f}\left[ \pm\left(\alpha_{1} \sigma\right)\right]$, where $\mathrm{V}_{1}$ and $V_{2}$ are constants, and $\mathrm{f}(\phi, \sigma)$ is an arbitrary function. We select the simplest form of the potential $\mathrm{V}(\phi, \sigma)=\mathrm{A}(\phi) \mathrm{B}(\sigma)$ :

$$
\mathrm{V}=\mathrm{V}_{0} \mathrm{e}^{-\lambda_{1} \phi-\lambda_{2} \sigma}
$$

where $\mathrm{V}_{0}$ is a constants and $\lambda_{1}$ and $\lambda_{2}$ are distinguishing parameters. This class of potential has been obtained by other methods; see, for instance, [27,32-35]. Therefore the time derivative of the momenta is simply $\dot{\Pi}_{\phi}=-\lambda_{1} \mathrm{U}$ and $\dot{\Pi}_{\sigma}=$ $-\lambda_{2} \mathrm{U}$, of which solutions are

$$
\begin{aligned}
& \Pi_{\phi}=-\frac{\lambda_{1}}{6} \Pi_{\Omega}+p_{\phi}, \\
& \Pi_{\sigma}=-\frac{\lambda_{2}}{6} \Pi_{\Omega}+p_{\sigma},
\end{aligned}
$$

where $\mathrm{p}_{\phi}$ and $\mathrm{p}_{\sigma}$ are integration constants. Henceforth we will employ this scheme in order to find analytic classic and quantum solutions.

\section{Classical Solutions}

We start from the Hamilton equations (13) in order to find relations between the scale factor and the scalar fields, such as

$$
\begin{aligned}
& \dot{\phi}=-24 \Pi_{\phi}=4 \lambda_{1} \Pi_{\Omega}-24 p_{\phi}=2 \lambda_{1} \dot{\Omega}-24 p_{\phi}, \\
& \dot{\sigma}=-24 \Pi_{\sigma}=4 \lambda_{2} \Pi_{\Omega}-24 p_{\sigma}=2 \lambda_{2} \dot{\Omega}-24 p_{\sigma},
\end{aligned}
$$

of which solutions are

$$
\begin{aligned}
& \phi=\phi_{1}+2 \lambda_{1} \Omega-24 p_{\phi} t, \\
& \sigma=\sigma_{1}+2 \lambda_{2} \Omega-24 p_{\sigma} t,
\end{aligned}
$$

where $\phi_{1}$ and $\sigma_{1}$ are integration constants, and they can be determined by suitable conditions. These expressions are indeed general relations, since they satisfy the Einstein-KleinGordon equations ((6)-(9)). Then by taking into account the constraint $\mathscr{H}=0$, we obtain the temporal dependence for $\Pi_{\Omega}(\mathrm{t})$ which allows us to construct a master equation:

$$
\frac{\mathrm{d} \Pi_{\Omega}}{\mathrm{m}_{1} \Pi_{\Omega}^{2}+\mathrm{m}_{2} \Pi_{\Omega}-\mathrm{m}_{3}}=\mathrm{dt},
$$

where the parameters $m_{i}, i=1,2,3$, are

$$
\begin{aligned}
& \mathrm{m}_{1}=2\left(3-\lambda_{1}^{2}-\lambda_{2}^{2}\right)=2\left(3-\lambda^{2}\right), \\
& \mathrm{m}_{2}=24\left[\lambda_{1} \mathrm{p}_{\phi}+\lambda_{2} \mathrm{p}_{\sigma}\right], \\
& \mathrm{m}_{3}=72\left[\mathrm{p}_{\phi}^{2}+\mathrm{p}_{\sigma}^{2}\right] .
\end{aligned}
$$

Subsequently by analyzing the parameter $\lambda^{2}=\lambda_{1}^{2}+\lambda_{2}^{2}$ we will obtain three different solutions.

3.1. Solution for $\lambda^{2}=3$. Having $\lambda^{2}=3$ implies that $\mathrm{m}_{1}=0$, so the integral to solve becomes

$$
\int \frac{\mathrm{d} \Pi_{\Omega}}{\mathrm{m}_{2} \Pi_{\Omega}-\mathrm{m}_{3}}=\int \mathrm{dt}
$$

then we parameterize $\lambda_{i}$ such as $\lambda_{1}=\sqrt{3(1-\epsilon)}, \lambda_{2}=$ $\sqrt{3 \epsilon}, \lambda^{2}=3$, where $\epsilon \in(0,1)$ measures the corresponding weight for each scalar field during inflation; so the constants (21) become

$$
\begin{aligned}
& \mathrm{m}_{2}=24 \sqrt{3(1-\epsilon)}\left[\mathrm{p}_{\phi}+\sqrt{\frac{\epsilon}{(1-\epsilon)}} \mathrm{p}_{\sigma}\right], \\
& \mathrm{m}_{3}=72\left[\mathrm{p}_{\phi}^{2}+\mathrm{p}_{\sigma}^{2}\right] .
\end{aligned}
$$


Thus $\Pi_{\Omega}(\mathrm{t})$ becomes

$$
\Pi_{\Omega}(\mathrm{t})=\frac{\mathrm{m}_{3}}{\mathrm{~m}_{2}}+\mathrm{c}_{1} \mathrm{e}^{\mathrm{bt}}
$$

where $c_{1}$ is an integration constant. Using the relations from (13) and after some algebra, the solutions of the set of variables $(\Omega, \phi, \sigma)$ and $\left(\Pi_{\phi}, \Pi_{\sigma}\right)$ are

$$
\begin{aligned}
\Omega= & \Omega_{0}+\frac{2 \mathrm{~m}_{3}}{\mathrm{~m}_{2}} \mathrm{t}+\frac{2 \mathrm{c}_{1}}{\mathrm{~m}_{2}} \mathrm{e}^{\mathrm{m}_{2} \mathrm{t}}, \\
\phi= & \phi_{0}+4 \sqrt{3(1-\epsilon)} \frac{\mathrm{m}_{3}}{\mathrm{~m}_{2}} \mathrm{t}-24 \mathrm{p}_{\phi} \mathrm{t} \\
& +\frac{4 \mathrm{c}_{1} \sqrt{3(1-\epsilon)}}{\mathrm{m}_{2}} \mathrm{e}^{\mathrm{m}_{2} \mathrm{t}}, \\
\sigma= & \sigma_{0}+4 \sqrt{3 \epsilon} \frac{\mathrm{m}_{3}}{\mathrm{~m}_{2}} \mathrm{t}-24 \mathrm{p}_{\sigma} \mathrm{t}+\frac{4 \mathrm{c}_{1} \sqrt{3 \epsilon}}{\mathrm{m}_{2}} \mathrm{e}^{\mathrm{m}_{2} \mathrm{t}}, \\
\Pi_{\phi}= & -\frac{\sqrt{3(1-\epsilon)}}{6}\left(\frac{\mathrm{m}_{3}}{\mathrm{~m}_{2}}+\mathrm{c}_{1} \mathrm{e}^{\mathrm{m}_{2} \mathrm{t}}\right)+\mathrm{p}_{\phi}, \\
\Pi_{\sigma}= & -\frac{\sqrt{3 \epsilon}}{6}\left(\frac{\mathrm{m}_{3}}{\mathrm{~m}_{2}}+\mathrm{c}_{1} \mathrm{e}^{\mathrm{m}_{2} \mathrm{t}}\right)+\mathrm{p}_{\sigma},
\end{aligned}
$$

where $\left(\Omega_{0}, \phi_{0}, \sigma_{0}\right)$ are all integration constants. In order to make the above results fulfill the EKG equations ((6)-(9)), all constants must satisfy that $144 \mathrm{~V}_{0}=\mathrm{m}_{2} \mathrm{c}_{1} \mathrm{e}^{-6 \Omega_{0}+\lambda_{1} \phi_{0}+\lambda_{2} \sigma_{0}}$. Finally the scale factor $\mathrm{A}(\mathrm{t})$ for this case is

$$
A=A_{0} e^{\left(2 m_{3} / m_{2}\right) t} \exp \left[\frac{2 c_{1}}{m_{2}} e^{m_{2} t}\right],
$$

where $\mathrm{A}_{0}=\mathrm{e}^{\Omega_{0}}$. Given that the scale factor is an exponential of an exponential function, it might exhibit a highly substantial growth.

3.2. Solution for $\lambda^{2}>3$. For this case $m_{1}=2\left(3-\lambda^{2}\right)<0$, so the integral to solve becomes

$$
\frac{d \Pi_{\Omega}}{-m_{1} \Pi_{\Omega}^{2}+m_{2} \Pi_{\Omega}-m_{3}}=d t
$$

where we include the minus sign in this equation, such the constant $\mathrm{m}_{1}=2\left(\lambda^{2}-3\right)=2 \beta>0$. Then we define $\omega^{2}=$ $\mathrm{m}_{2}^{2}-8 \beta \mathrm{m}_{3}$, so we change variable as $\mathrm{z}=4 \beta \Pi_{\Omega}-\mathrm{m}_{2}$ in order to integrate (31). Thus the solution to the momenta $\Pi_{\Omega}(t)$ becomes

$$
\Pi_{\Omega}=\frac{\mathrm{m}_{2}}{4 \beta}+\frac{\omega}{4 \beta} \tanh \left(\frac{\omega}{2} \mathrm{t}\right) .
$$

Using the relations from (13) and after some algebra, the solutions of the set of variables $(\Omega, \phi, \sigma)$ and $\left(\Pi_{\phi}, \Pi_{\sigma}\right)$ are

$$
\begin{aligned}
\Omega= & \Omega_{0}+\frac{\mathrm{m}_{2}}{2 \beta} \mathrm{t}+\frac{1}{\beta} \ln \left[\cosh \left(\frac{\omega}{2} \mathrm{t}\right)\right], \\
\phi= & \phi_{0}+\left(\lambda_{1} \frac{\mathrm{m}_{2}}{\beta}-24 \mathrm{p}_{\phi}\right) \mathrm{t} \\
& -\frac{2 \lambda_{1}}{\beta} \ln \left[\cosh \left(\frac{\omega}{2} \mathrm{t}\right)\right], \\
\sigma= & \sigma_{0}+\left(\lambda_{2} \frac{\mathrm{m}_{2}}{\beta}-24 \mathrm{p}_{\sigma}\right) \mathrm{t}-\frac{2 \lambda_{2}}{\beta} \ln \left[\cosh \left(\frac{\omega}{2} \mathrm{t}\right)\right], \\
\Pi_{\phi}= & -\frac{\lambda_{1}}{6}\left(\frac{\mathrm{m}_{2}}{4 \beta}+\frac{\omega}{4 \beta} \tanh \left(\frac{\omega}{2} \mathrm{t}\right)\right)+\mathrm{p}_{\phi}, \\
\Pi_{\sigma}= & -\frac{\lambda_{2}}{6}\left(\frac{\mathrm{m}_{2}}{4 \beta}+\frac{\omega}{4 \beta} \tanh \left(\frac{\omega}{2} \mathrm{t}\right)\right)+\mathrm{p}_{\sigma},
\end{aligned}
$$

where $\left(\Omega_{0}, \phi_{0}, \sigma_{0}\right)$ are all integration constants. In order to make the above results fulfill the EKG equations ((6)-(9)), all constants must satisfy that $1152 \beta \mathrm{V}_{0}=\omega^{2} \mathrm{e}^{\lambda_{1} \phi_{0}+\lambda_{2} \sigma_{0}+2 \beta \Omega_{0}}$. Finally the scale factor becomes

$$
A=A_{0} e^{\left(m_{2} / 2 \beta\right) t}\left[\cosh \left(\frac{\omega}{2} t\right)\right]^{1 / \beta},
$$

where $\mathrm{A}_{0}=e^{\Omega_{0}}$. For this case, given that $\beta>0$, one would expect that the scale factor grows slower than the previous case $\lambda^{2}=3$.

3.3. Solution When $\lambda^{2}<3$. For this case we modify the relation between the momenta equation (15), by changing the sign in the constants, $\left(\mathrm{p}_{\phi}, \mathrm{p}_{\sigma}, \mathrm{m}_{2}\right) \longrightarrow\left(-\mathrm{p}_{\phi},-\mathrm{p}_{\sigma},-\mathrm{m}_{2}\right)$, and $\mathrm{m}_{1}=2\left(3-\lambda^{2}\right)=2 \eta>0$; therefore the integral to solve becomes

$$
\frac{\mathrm{d} \Pi_{\Omega}}{2 \eta \Pi_{\Omega}^{2}-\mathrm{m}_{2} \Pi_{\Omega}-\mathrm{m}_{3}}=\mathrm{dt} .
$$

Thus $\Pi_{\Omega}(\mathrm{t})$ is

$$
\Pi_{\Omega}=\frac{1}{4 \eta}\left[\mathrm{m}_{2}-\alpha \operatorname{coth}\left(\frac{\alpha}{2} \mathrm{t}\right)\right]
$$

where $\alpha^{2}=\mathrm{m}_{2}^{2}+8 \eta \mathrm{m}_{3}$. Using the relations from (13) and after some algebra, the solutions of the set of variables $(\Omega, \phi, \sigma)$ and $\left(\Pi_{\phi}, \Pi_{\sigma}\right)$ are 


$$
\begin{aligned}
\Omega & =\Omega_{0}+\frac{\mathrm{m}_{2}}{2 \eta} \mathrm{t}+\ln \left[\operatorname{csch}\left(\frac{\alpha}{2} \mathrm{t}\right)\right]^{1 / \eta} \\
\phi & =\phi_{0}+\left(\lambda_{1} \frac{\mathrm{m}_{2}}{\eta}+24 \mathrm{p}_{\phi}\right) \mathrm{t}-\ln \left[\sinh \left(\frac{\alpha}{2} \mathrm{t}\right)\right]^{2 \lambda_{1} / \eta}, \\
\sigma & =\sigma_{0}+\left(\lambda_{2} \frac{\mathrm{m}_{2}}{\eta}+24 \mathrm{p}_{\sigma}\right) \mathrm{t}-\ln \left[\sinh \left(\frac{\alpha}{2} \mathrm{t}\right)\right]^{2 \lambda_{2} / \eta} \\
\Pi_{\phi} & =-\frac{1}{24}\left[\frac{\lambda_{1} \mathrm{~m}_{2}}{\eta}+24 \mathrm{p}_{\phi}\right]+\frac{\lambda_{1} \alpha}{24 \eta} \operatorname{coth}\left(\frac{\alpha}{2} \mathrm{t}\right) \\
\Pi_{\sigma} & =-\frac{1}{24}\left[\frac{\lambda_{2} \mathrm{~m}_{2}}{\eta}+24 \mathrm{p} \phi\right]+\frac{\lambda_{2} \alpha}{24 \eta} \operatorname{coth}\left(\frac{\alpha}{2} \mathrm{t}\right)
\end{aligned}
$$

where $\left(\Omega_{0}, \phi_{0}, \sigma_{0}\right)$ are integration constants. In order to fulfill the EKG equations ((6)-(9)), all constants must satisfy that $1152 \eta \mathrm{V}_{0}=\alpha^{2} \mathrm{e}^{\lambda_{1} \phi_{0}+\lambda_{2} \sigma_{0}-2 \eta \Omega_{0}}$. Finally the scale factor $\mathrm{A}=\mathrm{e}^{\Omega}$ becomes

$$
\mathrm{A}=\mathrm{A}_{0} \mathrm{e}^{\left(\mathrm{m}_{2} / 2 \eta\right) \mathrm{t}} \operatorname{csch}^{1 / \eta}\left(\frac{\alpha}{2} \mathrm{t}\right),
$$

where $\mathrm{A}_{0}=\mathrm{e}^{\Omega_{0}}$. For this case, given that $\eta>0$, one would expect the scale factor to grow in a similar way as the previous case $\lambda^{2}>3$.

3.4. Number of e-Folds. Inflation is characterised by the number of e-folds it expands during such period that corresponds to $\mathrm{A}_{\text {phys }}^{\prime \prime}>0$, where the primes represent the derivatives with respect to the cosmic time $t_{\text {phys }}$. The e-folding function $\mathrm{N}_{\mathrm{e}}=$ $\int \mathrm{dt}_{\text {phys }} \mathrm{H}\left(\mathrm{t}_{\text {phys }}\right)$ is described by $\mathrm{t}_{\text {phys }}$ : computing the integral from $t_{\text {phys }} *$ to $t_{\text {phys end }}$, where $t_{\text {phys }} *$ represents the time when the relevant cosmic microwave background (CMB) modes become superhorizon at 50-60 e-folds before inflation ends at $\mathrm{t}_{\text {phys end }}$; and $\mathrm{H}\left(\mathrm{t}_{\text {phys }}\right)=\mathrm{H}_{\text {phys }}=\mathrm{A}_{\text {phys }}^{\prime} / \mathrm{A}_{\text {phys }}$ is the Hubble parameter. Although, in our prescription we use a proper time $t$, we can evaluate the Hubble function in the corresponding gauge as $\mathrm{H}_{\text {phys }}=\dot{\Omega} / \mathrm{N}$.

At the end of inflation the expansion rate of the scale factor must be null which translates to $-\mathrm{H}_{\text {phys }}^{\prime}=\mathrm{H}_{\text {phys }}^{2}$ or $\ddot{\Omega}=2 \dot{\Omega}^{2}$. From here we can compute the time when inflation ends $\left(t_{\text {end }}\right)$ given each particular case. Table 1 shows the computation of the e-folding function $\mathrm{N}_{\mathrm{e}}$ and $\mathrm{t}_{\text {end }}$ for each case given by the $\lambda$ parameter.

\section{Quantum Solutions}

The Wheeler-DeWitt equation for this model is acquired by replacing $\Pi_{\mathrm{q}^{\mu}}=-\mathrm{i} \hbar \partial_{\mathrm{q}^{\mu}}$ in (12). The factor $\mathrm{e}^{-3 \Omega}$ may be factor ordered with $\widehat{\Pi}_{\Omega}$ in several forms. Hartle and Hawking [30] have suggested what might be called a semigeneral factor ordering, which in this case would order $\mathrm{e}^{-3 \Omega} \widehat{\Pi}_{\Omega}^{2}$ as

$$
-\mathrm{e}^{-(3-\mathrm{p}) \Omega} \partial_{\Omega} \mathrm{e}^{-\mathrm{p} \Omega} \partial_{\Omega}=-\mathrm{e}^{-3 \Omega} \partial_{\Omega}^{2}+\mathrm{pe}^{-3 \Omega} \partial_{\Omega},
$$

where $\mathrm{p}$ is any real constant that measures the ambiguity in the factor ordering for the variable $\Omega$; in the following we will assume such factor ordering for the Wheeler-DeWitt equation, which becomes

$$
\hbar^{2} \square \Psi+\hbar^{2} \mathrm{p} \frac{\partial \Psi}{\partial \Omega}-\mathrm{U}(\Omega, \phi, \sigma) \Psi=0,
$$

where $\square=-\partial^{2} / \partial \Omega^{2}+(1 / 12)\left(\partial^{2} / \partial \phi^{2}\right)+(1 / 12)\left(\partial^{2} / \partial \sigma^{2}\right)$ is the d'Alambertian in the coordinates $q^{\mu}=(\Omega, \phi, \sigma)$ and the potential is $\mathrm{U}=+24 \mathrm{~V}_{0} \mathrm{e}^{6 \Omega-\lambda_{1} \phi-\lambda_{2} \sigma}$. Then we transform the coordinates to obtain a potential that only depends on a single variable, employing the following transformation:

$$
\begin{aligned}
& \zeta=6 \Omega-\lambda_{1} \phi-\lambda_{2} \sigma, \\
& \kappa=\phi+\sigma, \\
& \eta=\phi-\sigma .
\end{aligned}
$$

Now we find the partial derivatives of $\psi$ with respect to the old coordinates $(\mathrm{a}, \phi, \sigma)$ but in terms of the new variables $(\zeta$, $\kappa, \eta)$

$$
\begin{aligned}
\frac{\partial \Psi}{\partial \Omega} & =\frac{\partial \Psi}{\partial \zeta} \frac{\partial \zeta}{\partial \Omega}+\frac{\partial \Psi}{\partial \kappa} \frac{\partial \kappa}{\partial \Omega}+\frac{\partial \Psi}{\partial \eta} \frac{\partial \eta}{\partial \Omega}=6 \frac{\partial \Psi}{\partial \zeta} \\
\frac{\partial \Psi}{\partial \phi} & =\frac{\partial \Psi}{\partial \zeta} \frac{\partial \zeta}{\partial \phi}+\frac{\partial \Psi}{\partial \kappa} \frac{\partial \kappa}{\partial \phi}+\frac{\partial \Psi}{\partial \eta} \frac{\partial \eta}{\partial \phi} \\
& =-\lambda_{1} \frac{\partial \Psi}{\partial \zeta}+\frac{\partial \Psi}{\partial \kappa}+\frac{\partial \Psi}{\partial \eta} \\
\frac{\partial \Psi}{\partial \sigma} & =\frac{\partial \Psi}{\partial \zeta} \frac{\partial \zeta}{\partial \sigma}+\frac{\partial \Psi}{\partial \kappa} \frac{\partial \kappa}{\partial \sigma}+\frac{\partial \Psi}{\partial \eta} \frac{\partial \eta}{\partial \sigma} \\
& =-\lambda_{2} \frac{\partial \Psi}{\partial \zeta}+\frac{\partial \Psi}{\partial \kappa}-\frac{\partial \Psi}{\partial \eta}
\end{aligned}
$$

from here we use these new relations in the quantum Hamiltonian density, obtaining

$$
\begin{aligned}
& 12 \hbar^{2}\left(\lambda_{1}^{2}+\lambda_{2}^{2}-3\right) \frac{\partial^{2} \Psi}{\partial \zeta^{2}}+24 \hbar^{2}\left(-\lambda_{1}+\lambda_{2}\right) \frac{\partial^{2} \Psi}{\partial \eta \partial \zeta} \\
& \quad+24 \hbar^{2} \frac{\partial^{2} \Psi}{\partial \eta^{2}}-24 \hbar^{2}\left(\lambda_{1}+\lambda_{2}\right) \frac{\partial^{2} \Psi}{\partial \kappa \partial \zeta}+24 \hbar^{2} \frac{\partial^{2} \Psi}{\partial \kappa^{2}} \\
& \quad-6 \hbar^{2} \mathrm{p} \frac{\partial \Psi}{\partial \zeta}-24 \mathrm{~V}_{0} \mathrm{e}^{\zeta} \Psi=0
\end{aligned}
$$

At this point, we propose the following ansatz, $\Psi=$ $\mathrm{e}^{(1 / \hbar)\left(c_{2} \kappa+c_{3} \eta\right)} \mathrm{G}(\zeta)$, where the parameters $c_{\mathrm{i}}$ are constants and $\mathrm{G}(\zeta)$ is a function to be determined. By introducing the aforementioned into (51) we obtain the following differential equation of the function $\mathrm{G}$ :

$$
\delta_{0} \frac{\mathrm{d}^{2} \mathrm{G}}{\mathrm{d} \zeta^{2}}+\alpha_{0} \frac{\mathrm{dG}}{\mathrm{d} \zeta}+\left(\beta_{0} \mathrm{e}^{\zeta}+\rho_{0}\right) \mathrm{G}=0
$$




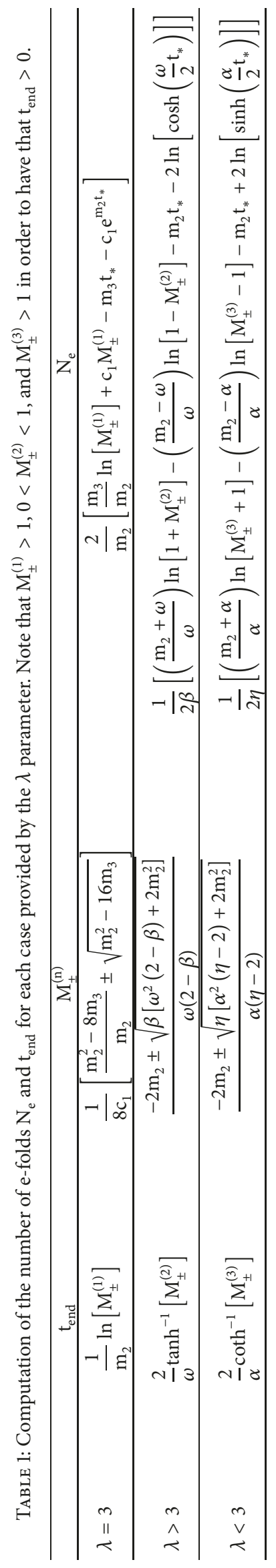


where the constants are

$$
\begin{aligned}
& \delta_{0}=12 \hbar^{2}\left(\lambda_{1}^{2}+\lambda_{2}^{2}-3\right), \\
& \alpha_{0}=-6 \hbar\left[4 \lambda_{1}\left(c_{2}+c_{3}\right)+4 \lambda_{2}\left(c_{2}-c_{3}\right)+\hbar \mathrm{p}\right], \\
& \beta_{0}=-24 V_{0}, \\
& \rho_{0}=24\left(c_{2}^{2}+c_{3}^{2}\right) .
\end{aligned}
$$

The solution of (52) is dependant on the value of constant $\delta_{0}$, which turns in three different cases, (I) $\delta=0$ implying that $\lambda_{1}^{2}+\lambda_{2}^{2}=3$, (II) $\delta<0$ implying that $\lambda_{1}^{2}+\lambda_{2}^{2}<3$, and (III) $\delta>0$ implying that $\lambda_{1}^{2}+\lambda_{2}^{2}>3$, which can be analyzed in two different cases.

4.1. Case $\delta=0$. For this case, (52) becomes

$$
\alpha_{0} \frac{\mathrm{dG}}{\mathrm{d} \zeta}+\left(\beta_{0} \mathrm{e}^{\zeta}+\rho_{0}\right) \mathrm{G}=0
$$

of which solution is

$$
\begin{aligned}
& \mathrm{G}(\zeta)=\mathrm{e}^{\mathrm{c}_{1} \zeta} \\
& \quad \cdot \exp \left[-\frac{4 \mathrm{~V}_{0}}{\hbar\left[4 \lambda_{1}\left(c_{2}+c_{3}\right)+4 \lambda_{2}\left(c_{2}-c_{3}\right)+\hbar \mathrm{p}\right]} e^{\zeta}\right], \\
& c_{1}=\frac{4\left(c_{2}^{2}+c_{3}^{2}\right)}{\hbar\left[4 \lambda_{1}\left(c_{2}+c_{3}\right)+4 \lambda_{2}\left(c_{2}-c_{3}\right)+\hbar \mathrm{p}\right]},
\end{aligned}
$$

and therefore, the corresponding wave function for this case becomes

$$
\begin{aligned}
& \Psi=\psi_{0} \mathrm{e}^{(1 / \hbar)\left(c_{1} \zeta+c_{2} \kappa+c_{3} \eta\right)} \exp \left[-c_{4} \mathrm{e}^{\zeta}\right], \\
& c_{4}=\frac{4 \mathrm{~V}_{0}}{\hbar\left[4 \lambda_{1}\left(c_{2}+c_{3}\right)+4 \lambda_{2}\left(c_{2}-c_{3}\right)+\hbar \mathrm{p}\right]} .
\end{aligned}
$$

Note that wave function has a damping behavior with respect to the scale factor, which is a required feature.

4.2. Case $\delta \neq 0$. For this case, (52) becomes, which is similar to that in [36],

$$
\begin{aligned}
\mathrm{y}^{\prime \prime}+a \mathrm{y}^{\prime}+\left(\mathrm{be} \mathrm{ex}^{\kappa \mathrm{x}}+\mathrm{c}\right) \mathrm{y} & =0, \\
\mathrm{y} & =\mathrm{e}^{-\mathrm{ax} / 2} \mathrm{Z}_{\nu}\left(\frac{2 \sqrt{\mathrm{b}}}{\kappa} \mathrm{e}^{\kappa \mathrm{x} / 2}\right),
\end{aligned}
$$

where $\mathrm{Z}_{\nu}$ is the Bessel function and $v=\sqrt{a^{2}-4 c} / \kappa$ is the corresponding order, and its relations are

$$
\begin{aligned}
\mathrm{a} & =\frac{\alpha_{0}}{\delta_{0}}=-\frac{4 \lambda_{1}\left(\mathrm{c}_{2}+\mathrm{c}_{3}\right)+4 \lambda_{2}\left(\mathrm{c}_{2}-\mathrm{c}_{3}\right)+\hbar \mathrm{p}}{\hbar\left(\lambda_{1}^{2}+\lambda_{2}^{2}-3\right)}, \\
\mathrm{b} & =\frac{\beta_{0}}{\alpha_{0}} \\
& = \begin{cases}-\frac{2 \mathrm{~V}_{0}}{\hbar^{2}\left(\lambda_{1}^{2}+\lambda_{2}^{2}-3\right)}, & \text { when } \lambda_{1}^{2}+\lambda_{2}^{2}>3 \\
\frac{2 \mathrm{~V}_{0}}{\hbar^{2}\left(\lambda_{1}^{2}+\lambda_{2}^{2}-3\right)}, & \text { when } \lambda_{1}^{2}+\lambda_{2}^{2}<3\end{cases} \\
\mathrm{c} & =\frac{\rho_{0}}{\alpha_{0}}=\frac{2\left(c_{2}^{2}+c_{3}^{2}\right)}{\hbar^{2}\left(\lambda_{1}^{2}+\lambda_{2}^{2}-3\right)}, \\
\kappa & =1,
\end{aligned}
$$

of which, according to the constant $\mathrm{b}$, the solution to the function $\mathrm{G}$ becomes

$$
\begin{aligned}
\mathrm{G}(\zeta) & =\mathrm{e}^{\left(\left(4 \lambda_{1}\left(\mathrm{c}_{2}+\mathrm{c}_{3}\right)+4 \lambda_{2}\left(\mathrm{c}_{2}-c_{3}\right)+\hbar \mathrm{p}\right) / 2 \hbar\left(\lambda_{1}^{2}+\lambda_{2}^{2}-3\right)\right) \zeta} \mathrm{K}_{\nu}\left(\frac{2}{\hbar}\right. \\
\cdot & \left.\sqrt{\frac{2 \mathrm{~V}_{0}}{\lambda_{1}^{2}+\lambda_{2}^{2}-3}} \mathrm{e}^{\zeta / 2}\right), \quad \lambda_{1}^{2}+\lambda_{2}^{2}>3 \\
\mathrm{G}(\zeta) & =\mathrm{e}^{-\left(\left(4 \lambda_{1}\left(\mathrm{c}_{2}+\mathrm{c}_{3}\right)+4 \lambda_{2}\left(\mathrm{c}_{2}-\mathrm{c}_{3}\right)+\hbar \mathrm{p}\right) / 2 \hbar\left(3-\lambda_{1}^{2}+\lambda_{2}^{2}\right)\right) \zeta} \mathrm{J}_{\nu}\left(\frac{2}{\hbar}\right. \\
\cdot & \left.\sqrt{\frac{2 \mathrm{~V}_{0}}{3-\lambda_{1}^{2}+\lambda_{2}^{2}}} \mathrm{e}^{\zeta / 2}\right), \quad \lambda_{1}^{2}+\lambda_{2}^{2}<3 .
\end{aligned}
$$

and the resulting wave functions are

$$
\begin{aligned}
& \Psi=\mathrm{e}^{\mathrm{c}_{5} \zeta+c_{2} \kappa+c_{3} \eta} \mathrm{K}_{\nu}\left(\frac{2}{\hbar} \sqrt{\frac{2 \mathrm{~V}_{0}}{\lambda_{1}^{2}+\lambda_{2}^{2}-3}} \mathrm{e}^{\zeta / 2}\right), \\
& \Psi=\mathrm{e}^{-\mathrm{c}_{6} \zeta+c_{2} \kappa+c_{3} \eta} J_{\nu}\left(\frac{2}{\hbar} \sqrt{\frac{2 \mathrm{~V}_{0}}{3-\lambda_{1}^{2}+\lambda_{2}^{2}}} \mathrm{e}^{\zeta / 2}\right), \\
& \lambda_{1}^{2}>3 \\
& \lambda_{2}^{2}<3 .
\end{aligned}
$$

where the constants are

$$
\begin{aligned}
& c_{5}=\frac{4 \lambda_{1}\left(c_{2}+c_{3}\right)+4 \lambda_{2}\left(c_{2}-c_{3}\right)+\hbar p}{2 \hbar\left(\lambda_{1}^{2}+\lambda_{2}^{2}-3\right)}, \\
& c_{6}=\frac{4 \lambda_{1}\left(c_{2}+c_{3}\right)+4 \lambda_{2}\left(c_{2}-c_{3}\right)+\hbar p}{2 \hbar\left(3-\lambda_{1}^{2}+\lambda_{2}^{2}\right)} .
\end{aligned}
$$

Whilst $c_{5}<0$ and $c_{6}>0$, the wave functions ((61), (62)) will remain suppressed by the growth of the scale factor, yielding an expected damped wave function. 


\section{Conclusions}

We studied a flat Friedmann-Robertson-Walker (FRW) multiscalar field cosmological model. We introduce the corresponding Einstein-Klein-Gordon (EKG) system of equations and the associated Hamiltonian density. Exact solutions to the EKG system are derived by means of Hamilton's approach where a particular scalar potential of the form $\mathrm{V}=\mathrm{V}_{0} \mathrm{e}^{-\lambda_{1} \phi-\lambda_{2} \sigma}$ was utilized, which gave rise to different cases dependant of the free parameter $\lambda$, for which the scalar fields, the scale factor, and the e-folding function were found. The Hamiltonian density was employed in order to compute the Wheeler-DeWitt (WDW) equation, which was solved by means of a change of variables. An ansatz for the wave function was proposed which in turn allowed us to find the exact form of the generic function and its constants which was composed by the aforementioned in terms of the free parameter $\lambda$. We found the model to be rather simple and its solutions to be quite interesting for a model building inflation.

\section{Data Availability}

No data were used to support this study and all required information to arrive to the findings of this work is included within the article.

\section{Conflicts of Interest}

The authors declare that they have no conflicts of interest.

\section{Acknowledgments}

This work was partially supported by CONACYT 167335 and 179881 grants and PROMEP grants UGTO-CA-3. Rafael Hernández-Jiménez acknowledges CONACYT for financial support. This work is part of the collaboration within the Instituto Avanzado de Cosmología. Many calculations were done by Symbolic Program REDUCE 3.8.

\section{References}

[1] A. H. Guth, "Inflationary universe: a possible solution to the horizon and flatness problems," Physical Review D: Particles, Fields, Gravitation and Cosmology, vol. 23, no. 2, pp. 347-356, 1981.

[2] A. D. Linde, "A new inflationary universe scenario: a possible solution of the horizon, flatness, homogeneity, isotropy and primordial monopole problems," Physics Letters B, vol. 108, no. 6, pp. 389-393, 1982.

[3] J. D. Barrow and M. S. Turner, "Inflation in the Universe," Nature, vol. 292, no. 5818, pp. 35-38, 1981.

[4] A. A. Starobinsky, "A new type of isotropic cosmological models without singularity," Physics Letters B, vol. 91, no. 1, pp. 99-102, 1980.

[5] A. A. Starobinsky, "Spectrum of relict gravitational radiation and the early state of the universe," JETP Lett, vol. 30, no. 682, 1979.

[6] V. F. Mukhanov and G. V. Chibisov, "Quantum Fluctuations and a Nonsingular Universe," JETP Lett, vol. 33, no. 532, 1981.
[7] H. Kodama and M. Sasaki, "Cosmological perturbation theory," Progress of Theoretical and Experimental Physics Supplement, vol. 78, pp. 1-166, 1984.

[8] B. A. Bassett, S. Tsujikawa, and D. Wands, "Inflation dynamics and reheating," Reviews of Modern Physics, vol. 78, no. 2, pp. 537-589, 2006.

[9] P. A. R. Ade and Planck Collaboration, "Planck 2018 results. X. Constraints on inflation," 2018, https://arxiv.org/abs/1807.06211.

[10] J. D. Barrow, "Slow-roll inflation in scalar-tensor theories," Physical Review D: Particles, Fields, Gravitation and Cosmology, vol. 51, p. 2729, 1995.

[11] A. R. Liddle and R. J. Scherrer, "Classification of scalar field potentials with cosmological scaling solutions," Physical Review D: Particles, Fields, Gravitation and Cosmology, vol. 59, no. 2, Article ID 023509, 7 pages, 1998.

[12] P. G. Ferreira and M Joyce, "Cosmology with a primordial scaling field," Phys. Rev. D, vol. 58, Article ID 023503, 1998.

[13] E. J. Copeland, M. Sami, and S. Tsujikawa, "Dynamics of dark energy," International Journal of Modern Physics D: Gravitation, Astrophysics, Cosmology, vol. 15, no. 11, pp. 1753-1935, 2006.

[14] E. J. Copeland, A. R. Liddle, and D. Wands, "Exponential potentials and cosmological scaling solutions," Physical Review D: Particles, Fields, Gravitation and Cosmology, vol. 57, no. 8, pp. 4686-4690, 1998.

[15] T. Barreiro, E. J. Copeland, and N. J. Nunes, "Quintessence arising from exponential potentials," Physical Review D: Particles, Fields, Gravitation and Cosmology, vol. 61, no. 12, 2000.

[16] G. Calcagni and A. R. Liddle, "Stability of multifield cosmological solutions," Physical Review D: Particles, Fields, Gravitation and Cosmology, vol. 77, no. 2, 023522, 6 pages, 2008.

[17] D. Sáez-Gómez, Scalar-Tensor theories and current Cosmology, Problems of Modern Cosmology, 2008, https://arxiv.org/abs/ 0812.1980.

[18] M. Capone, C. Rubano, and P. Scudellaro, "Slow rolling, inflation, and quintessence," EPL (Europhysics Letters), vol. 73, no. 1, pp. 149-155, 2006.

[19] E. W. Kolb and M. S. Turner, The Early Universe, AddisonWesley Publishing co., Illinois, USA, 1998.

[20] A. A. Coley and R. J. van den Hoogen, "Dynamics of multiscalar-field cosmological models and assisted inflation," Physical Review D: Particles, Fields, Gravitation and Cosmology, vol. 62, no. 2, 023517, 11 pages, 2000.

[21] E. J. Copeland, A. Mazumdar, and N. J. Nunes, "Generalized assisted inflation," Physical Review D: Particles, Fields, Gravitation and Cosmology, vol. 60, no. 8, 1999.

[22] S. Yokoyama, T. Suyama, and T. Tanaka, "Primordial nonGaussianity in multiscalar inflation," Physical Review D: Particles, Fields, Gravitation and Cosmology, vol. 77, no. 8, 2008.

[23] T. Chiba and M. Yamaguchi, "Extended slow-roll conditions and primordial fluctuations: multiple scalar fields and generalized gravity," Journal of Cosmology and Astroparticle Physics, vol. 2009, no. 01, pp. 019-019, 2009.

[24] A. R. Liddle, A. Mazumdar, and F. E. Schunck, "Assisted inflation," Physical Review D: Particles, Fields, Gravitation and Cosmology, vol. 58, no. 6, 1998.

[25] M. P. DeCross, D. I. Kaiser, A. Prabhu, C. Prescod-Weinstein, and E. I. Sfakianakis, "Preheating after multifield inflation with nonminimal couplings. I. Covariant formalism and attractor behavior," Physical Review D: Particles, Fields, Gravitation and Cosmology, vol. 97, no. 2, 2018. 
[26] S. C. Hotinli, J. Frazer, A. H. Jaffe, J. Meyers, L. C. Price, and E. R. Tarrant, "Effect of reheating on predictions following multiplefield inflation," Physical Review D: Particles, Fields, Gravitation and Cosmology, vol. 97, no. 2, 2018.

[27] J. Socorro and O. E. Nuñez, "Scalar potentials with multi-scalar fields from quantum cosmology and supersymmetric quantum mechanics," The European Physical Journal Plus, vol. 132, no. 168, 2017, https://arxiv.org/abs/1702.00478.

[28] G. W. Gibbons and L. P. Grishchuk, "What is a typical wave function for the universe?” Nuclear Physics B, vol. 313, no. 3, pp. 736-748, 1989.

[29] L. Z. Fang and R. Ruffini, Eds., Quantum cosmology, vol. 3 of Advanced Series in Astrophysics and Cosmology, World Scientific Publishing Co., Inc., Teaneck, NJ, USA, 1987.

[30] J. B. Hartle and S. W. Hawking, "Wave function of the Universe," Physical Review D, vol. 28, no. 2960, 1983.

[31] S. W. Hawking, "The quantum state of the universe," Nuclear Physics. B. Theoretical, Phenomenological, and Experimental High Energy Physics. Quantum Field Theory and Statistical Systems, vol. 239, no. 1, pp. 257-276, 1984.

[32] W. Guzmán, M. Sabido, J. Socorro et al., "Scalar potentials out of canonical quantum cosmology," International Journal of Modern Physics D: Gravitation, Astrophysics, Cosmology, vol. 16, no. 4, pp. 641-653, 2007.

[33] J. Socorro and M. D’oleire, "Inflation from supersymmetric quantum cosmology," Physical Review D, vol. 82, no. 4, Article ID 044008, pp. 1-7, 2010.

[34] J. Socorro, P. Romero, L. O. Pimentel, and M. Aguero, "Quintom potentials from quantum cosmology using the FRW cosmological model," International Journal of Theoretical Physics, vol. 52, no. 8, pp. 2722-2734, 2013.

[35] J. Socorro, M. Sabido, W. Ramírez et al., "Inflación cosmológica vista desde la mecánica cuántica supersimétrica," Notabilis Scientia, 2013.

[36] V. F. Zaitsev and A. D. Polyanin, Handbook of Exact Solutions for Ordinary Differential Equations, Taylor \& Francis Editorial, 2002. 


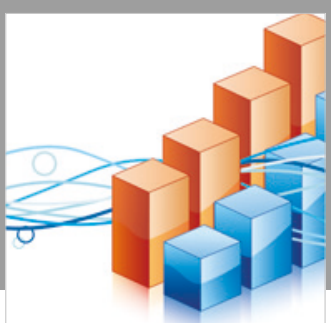

Advances in

Operations Research

\section{-n-m}
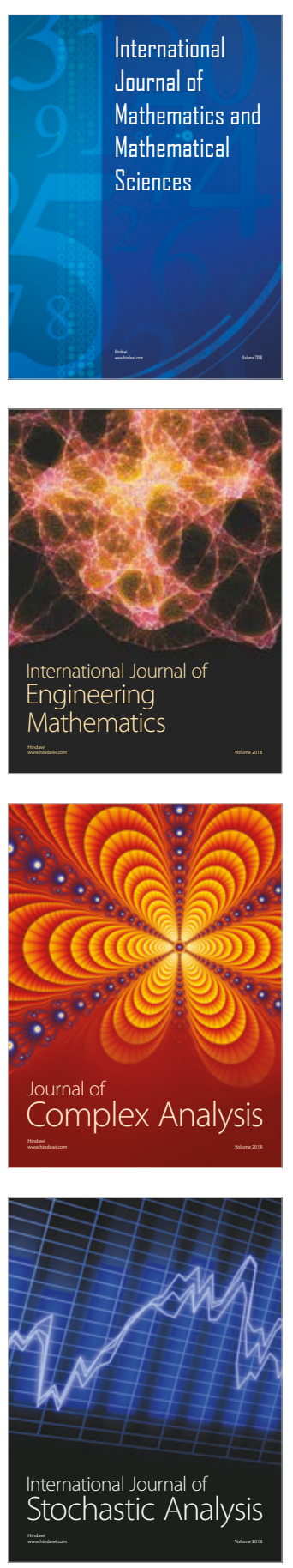
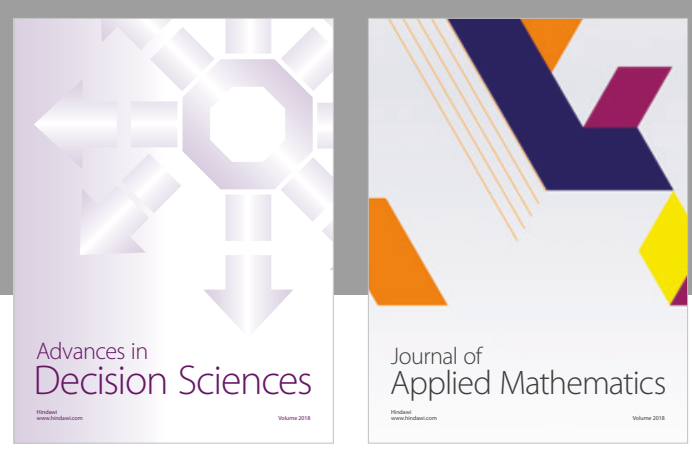

Journal of

Applied Mathematics
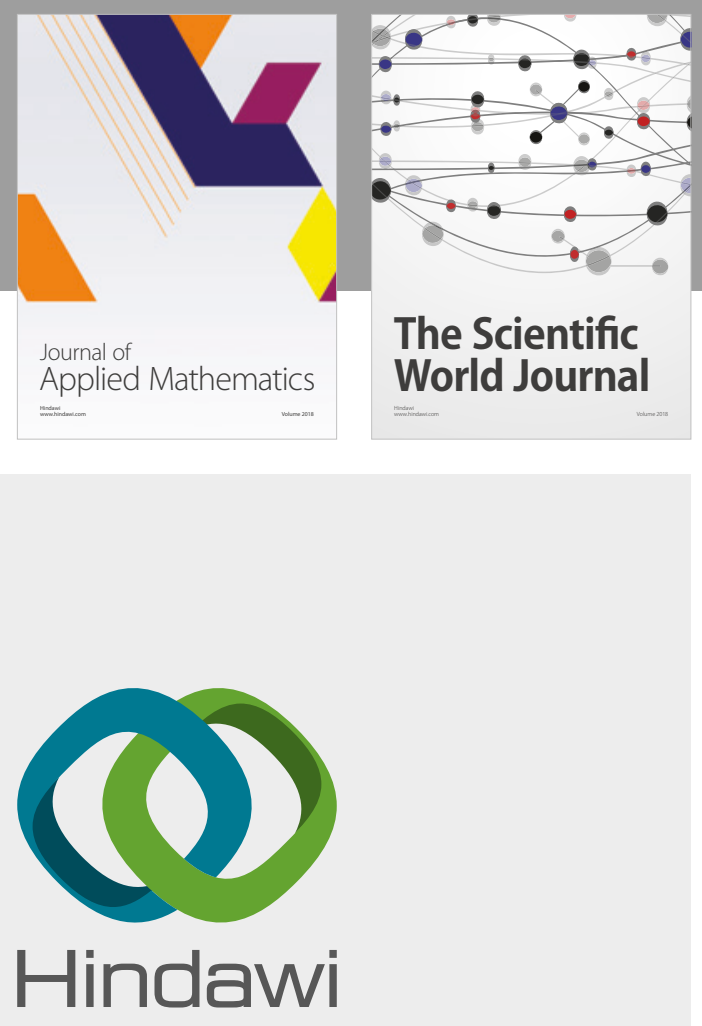

Submit your manuscripts at

www.hindawi.com

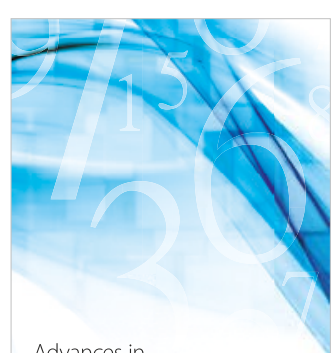

Advances in
Numerical Analysis
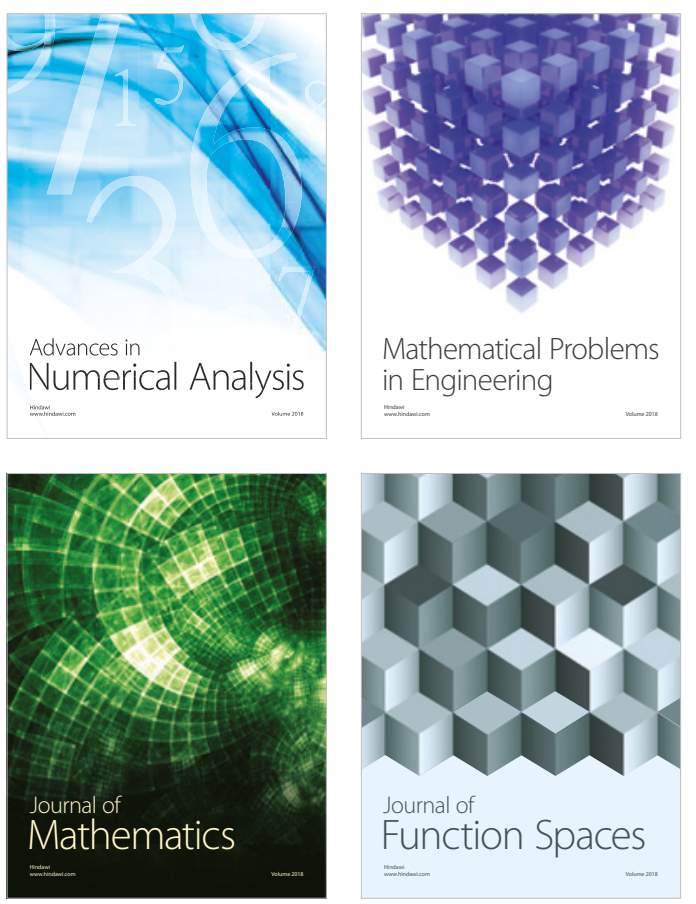

Mathematical Problems in Engineering

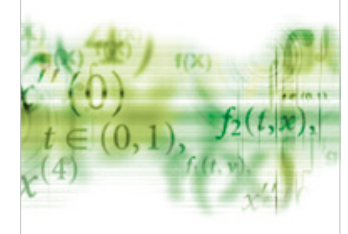

International Journal of

Differential Equations

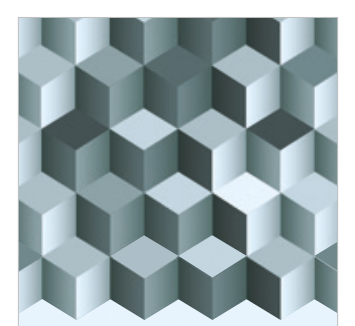

Journal of

Function Spaces

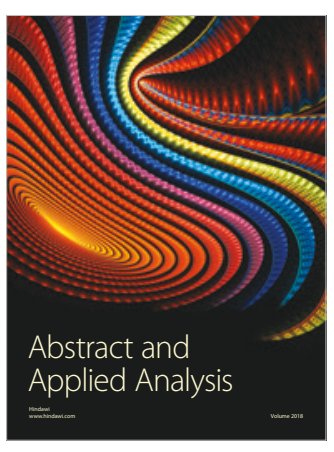

The Scientific

World Journal

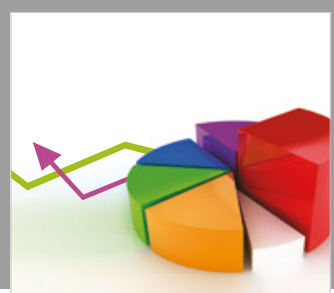

Journal of

Probability and Statistics
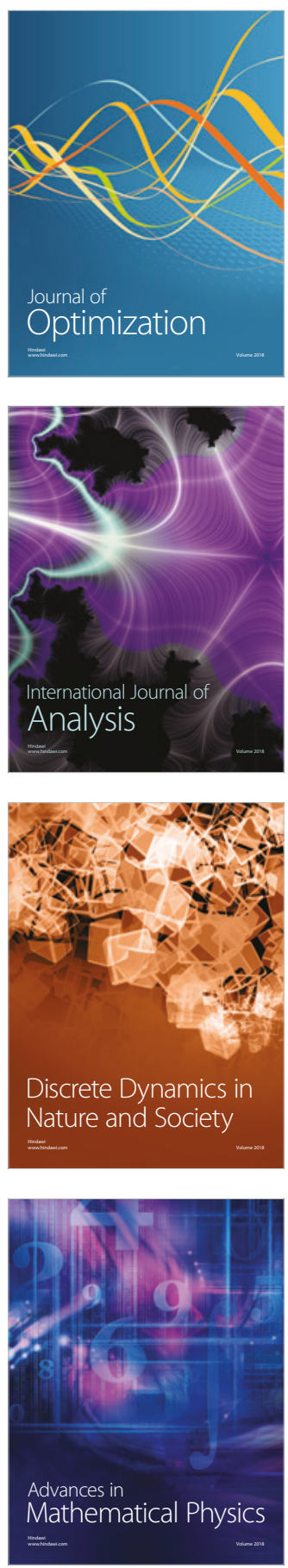